\title{
Generating Sets of the Complete Semigroups of Binary Relations Defined by Semilattices of the Class $\Sigma_{2}(X, 4)$
}

\author{
Bariş Albayrak¹, Omar Givradze², Guladi Partenadze² \\ ${ }^{1}$ Department of Banking and Finance, School of Applied Sciences, Çanakkale Onsekiz Mart University, Çanakkale, Turkey \\ ${ }^{2}$ Shota Rustaveli State University, Batumi, Georgia \\ Email: balbayrak77@gmail.com, omari@mail.ru, guladi@gmail.com
}

How to cite this paper: Albayrak, B., Givradze, O. and Partenadze, G. (2018) Generating Sets of the Complete Semigroups of Binary Relations Defined by Semilattices of the Class $\Sigma_{2}(X, 4)$. Applied Mathematics, 9, 17-27.

https://doi.org/10.4236/am.2018.91002

Received: December 11, 2017

Accepted: January 16, 2018

Published: January 19, 2018

Copyright $\odot 2018$ by authors and Scientific Research Publishing Inc. This work is licensed under the Creative Commons Attribution International License (CC BY 4.0).

http://creativecommons.org/licenses/by/4.0/

\begin{abstract}
In this paper, we have studied generating sets of the complete semigroups defined by $X$-semilattices of the class $\Sigma_{2}(X, 4)$.
\end{abstract}

\section{Keywords}

Semigroup, Semilattice, Binary Relations, Idempotent Elements

\section{Introduction}

Let $X$ be an arbitrary nonempty set and $D$ be a nonempty set of subsets of the set $X$. If $D$ is closed under the union, then $D$ is called a complete $X$-semilattice of unions. The union of all elements of the set $D$ is denoted by the symbol $\breve{D}$.

Let $B_{X}$ be the set of all binary relations on $X$. It is well known that $B_{X}$ is a semigroup.

Let $f$ be an arbitrary mapping from $X$ into $D$. Then we denote a binary relation $\alpha_{f}=\bigcup_{x \in X}(\{x\} \times f(x))$ for each $f$. The set of all such binary relations is denoted by $B_{X}(D)$. It is easy to prove that $B_{X}(D)$ is a semigroup with respect to the product operation of binary relations. This semigroup $B_{X}(D)$ is called a complete semigroup of binary relations defined by an $X$-semilattice of unions $D$. This structure was comprehensively investigated in Diasamidze [1] and [2]. We assume that $t, y \in X, Y \subseteq X, \alpha \in B_{X}, T \in D$ and $\varnothing \neq D^{\prime} \subseteq D$. Then we denote following sets

$$
y \alpha=\{x \in X \mid y \alpha x\}, Y \alpha=\bigcup_{y \in Y} y \alpha,
$$




$$
\begin{aligned}
& V(D, \alpha)=\{Y \alpha \mid Y \in D\}, X^{*}=\{Y \mid \varnothing \neq Y \subseteq X\} \\
& Y_{T}^{\alpha}=\{y \in X \mid y \alpha=T\}, V\left(X^{*}, \alpha\right)=\{Y \alpha \mid \varnothing \neq Y \subseteq X\} \\
& D_{t}=\left\{Z^{\prime} \in D \mid t \in Z^{\prime}\right\}, \quad B_{0}=\left\{\alpha \in B_{X}(D) \mid V\left(X^{*}, \alpha\right)=D\right\}
\end{aligned}
$$

Let $D=\left\{\breve{D}, Z_{1}, Z_{2}, \cdots, Z_{m-1}\right\} \quad$ be finite $X$-semilattice of unions and $C(D)=\left\{P_{0}, P_{1}, P_{2}, \cdots, P_{m-1}\right\}$ be the family of pairwise nonintersecting subsets of $X$. If $\varphi=\left(\begin{array}{cccc}\breve{D} & Z_{1} & \cdots & Z_{m-1} \\ P_{0} & P_{1} & \cdots & P_{m-1}\end{array}\right)$ is a mapping from $D$ on $C(D)$, then the equalities $\breve{D}=P_{0} \cup P_{1} \cup P_{2} \cup \cdots \cup P_{m-1}$ and $Z_{i}=P_{0} \cup \bigcup_{T \in D \backslash D_{Z}} \varphi(T)$ are valid. These equalities are called formal.

Let $D$ be a complete $X$-semilattice of unions $\alpha \in B_{X}$. Then a representation of a binary relation $\alpha$ of the form $\alpha=\bigcup_{T \in V\left(X^{*}, \alpha\right)}\left(Y_{T}^{\alpha} \times T\right)$ is called quasinormal.

Let $P_{0}, P_{1}, P_{2}, \cdots, P_{m-1}$ be parameters in the formal equalities, $\beta \in B_{X}(D), \bar{\beta}_{2}$ be mapping from $X \backslash \breve{D}$ to $D$. Then $\bar{\beta}=\bigcup_{i=0}^{m-1}\left(P_{i} \times \bigcup_{t \in P_{i}} t \beta\right) \cup \bigcup_{t^{\prime} \in X \backslash \breve{D}}\left(\left\{t^{\prime}\right\} \times \bar{\beta}_{2}\left(t^{\prime}\right)\right)$ is called subquasinormal represantation of $\beta$. It can be easily seen that the following statements are true.

a) $\bar{\beta} \in B_{X}(D)$.

b) $\bigcup_{i=0}^{m-1}\left(P_{i} \times \bigcup_{t \in P_{i}} t \beta\right) \subseteq \beta$ and $\beta=\bar{\beta}$ for some $\bar{\beta}_{2}$.

c) Subquasinormal represantation of $\beta$ is quasinormal.

d) $\bar{\beta}_{1}=\left(\begin{array}{cccc}P_{0} & P_{1} & \cdots & P_{m-1} \\ P_{0} \bar{\beta} & P_{1} \bar{\beta} & \cdots & P_{m-1} \bar{\beta}\end{array}\right)$ is mapping from $C(D)$ on $D \cup\{\varnothing\}$.

$\bar{\beta}_{1}$ and $\bar{\beta}_{2}$ are respectively called normal and complement mappings for $\beta$.

Let $\alpha \in B_{X}(D)$. If $\alpha \neq \delta \circ \beta$ for all $\delta, \beta \in B_{X}(D) \backslash\{\alpha\}$ then $\alpha$ is called external element. Every element of the set $B_{0}=\left\{\alpha \in B_{X}(D) \mid V\left(X^{*}, \alpha\right)=D\right\}$ is an external element of $B_{X}(D)$.

Theorem 1. [1] Let $X$ be a finite set and $\alpha, \beta \in B_{X}(D)$. If $\bar{\beta}$ is subquasinormal representation of $\beta$ then $\alpha \circ \beta=\alpha \circ \bar{\beta}$.

Corollary 1. [1] Let $\tilde{B}^{\prime} \subseteq \tilde{B} \subseteq B_{X}(D)$. If $\alpha \neq \delta \circ \bar{\beta}$ for $\alpha \in \tilde{B}^{\prime}, \delta \in \tilde{B} \backslash\{\alpha\}$, $\bar{\beta} \in \tilde{B} \backslash\{\alpha\}$ and subquasinormal representation of $\beta \in \tilde{B} \backslash\{\alpha\}$ then $\alpha \neq \delta \circ \beta$.

It is known that the set of all external elements is subset of any generating set of $B_{X}(D)$ in [3].

\section{Results}

In this work by symbol $\Sigma_{2.2}(X, 4)$ we denote all semilattices $D=\left\{Z_{3}, Z_{2}, Z_{1}, \breve{D}\right\}$ of the class $\Sigma_{2}(X, 4)$ which the intersection of minimal elements $Z_{3} \cap Z_{2}=\varnothing$. This semilattices graphic is given in Figure 1 . By using formal equalities, we have $Z_{3} \cap Z_{2}=P_{0}=\varnothing$. So, the formal equalities of the semilattice $D$ has a form 


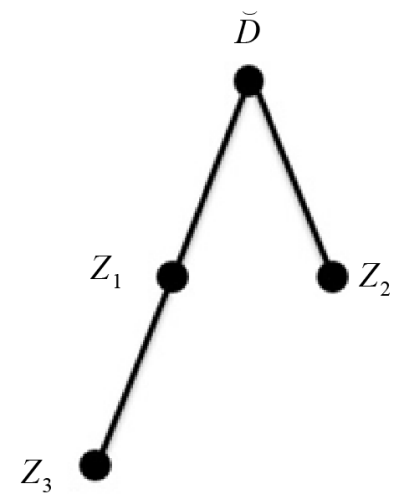

Figure 1. Graphic of semilattice $D=\left\{Z_{3}, Z_{2}, Z_{1}, D\right\}$ which the intersection of minimal elements $Z_{3} \cap Z_{2}=\varnothing$.

$$
\begin{aligned}
& \breve{D}=P_{1} \cup P_{2} \cup P_{3} \\
& Z_{1}=P_{2} \cup P_{3} \\
& Z_{2}=P_{1} \cup P_{3} \\
& Z_{3}=P_{2}
\end{aligned}
$$

Let $\delta, \bar{\beta} \in B_{X}(D)$. If quasinormal representation of binary relation $\delta$ has a form $\delta=\left(Y_{3}^{\delta} \times Z_{3}\right) \cup\left(Y_{2}^{\delta} \times Z_{2}\right) \cup\left(Y_{1}^{\delta} \times Z_{1}\right) \cup\left(Y_{0}^{\delta} \times \breve{D}\right)$ then

$$
\delta \circ \bar{\beta}=\left(Y_{3}^{\delta} \times Z_{3} \bar{\beta}\right) \cup\left(Y_{2}^{\delta} \times Z_{2} \bar{\beta}\right) \cup\left(Y_{1}^{\delta} \times Z_{1} \bar{\beta}\right) \cup\left(Y_{0}^{\delta} \times \breve{D} \bar{\beta}\right)
$$

We denote the set

$$
\begin{aligned}
& B_{32}=\left\{\alpha \in B_{X}(D) \mid V\left(X^{*}, \alpha\right)=\left\{Z_{3}, Z_{2}, \breve{D}\right\}\right\} \\
& B_{21}=\left\{\alpha \in B_{X}(D) \mid V\left(X^{*}, \alpha\right)=\left\{Z_{2}, Z_{1}, \breve{D}\right\}\right\} \\
& B_{31}=\left\{\alpha \in B_{X}(D) \mid V\left(X^{*}, \alpha\right)=\left\{Z_{3}, Z_{1}\right\}\right\} \\
& \tilde{B}_{32}=\left\{\alpha \in B_{32} \mid \alpha=\left(Y_{3}^{\alpha} \times Z_{3}\right) \cup\left(Y_{2}^{\alpha} \times Z_{2}\right), Y_{3}^{\alpha} \cup Y_{2}^{\alpha}=X, Y_{3}^{\alpha} \cap Y_{2}^{\alpha}=\varnothing\right\} \\
& \tilde{B}_{21}=\left\{\alpha \in B_{21} \mid \alpha=\left(Y_{2}^{\alpha} \times Z_{2}\right) \cup\left(Y_{1}^{\alpha} \times Z_{1}\right), Y_{2}^{\alpha} \cup Y_{1}^{\alpha}=X, Y_{2}^{\alpha} \cap Y_{1}^{\alpha}=\varnothing\right\}
\end{aligned}
$$

It is easy to see that

$$
B_{0} \cap B_{32}=B_{0} \cap B_{21}=B_{0} \cap B_{31}=B_{21} \cap B_{32}=B_{31} \cap B_{32}=B_{21} \cap B_{31}=\varnothing .
$$

Lemma 2. Let $D=\left\{Z_{3}, Z_{2}, Z_{1}, \breve{D}\right\} \in \Sigma_{2.2}(X, 4)$. Then following statements are true for the sets $B_{0}, B_{32}, \tilde{B}_{32}$.

a) If $\alpha=\left(Y_{3}^{\alpha} \times Z_{3}\right) \cup\left(Y_{1}^{\alpha} \times Z_{1}\right) \cup\left(Y_{0}^{\alpha} \times \breve{D}\right)$ for some $Y_{3}^{\alpha}, Y_{1}^{\alpha}, Y_{0}^{\alpha} \notin \varnothing$, then $\alpha$ is product of some elements of the set $B_{0}$.

b) If $\beta_{0}=\left(Z_{3} \times Z_{3}\right) \cup\left(\left(X \backslash Z_{3}\right) \times Z_{2}\right)$, then $\left(B_{0} \circ \beta_{0}\right) \cup \tilde{B}_{32}=B_{32}$.

c) If $\sigma_{1}=\left(Z_{2} \times Z_{2}\right) \cup\left(\left(X \backslash Z_{2}\right) \times Z_{1}\right)$, then $\left(B_{0} \circ \sigma_{1}\right) \cup \tilde{B}_{21}=B_{21}$.

d) If $\sigma_{1}=\left(Z_{2} \times Z_{2}\right) \cup\left(\left(X \backslash Z_{2}\right) \times Z_{1}\right)$, then $B_{32} \circ \sigma_{1}=B_{21}$.

e) If $\sigma_{0}=\left(Z_{3} \times Z_{3}\right) \cup\left(\left(X \backslash Z_{3}\right) \times Z_{1}\right)$, then $B_{32} \circ \sigma_{0}=B_{31}$.

f) Every element of the set $B_{32}$ is product of elements of the set $B_{0} \cup \tilde{B}_{32}$.

g) Every element of the set $B_{21}$ is product of elements of the set 
$B_{0} \cup \tilde{B}_{32} \cup\left\{\sigma_{1}\right\}$.

Proof. It will be enough to show only $a, b$ and $g$. The rest can be similarly seen.

a. Let $\alpha=\left(Y_{3}^{\alpha} \times Z_{3}\right) \cup\left(Y_{1}^{\alpha} \times Z_{1}\right) \cup\left(Y_{0}^{\alpha} \times \breve{D}\right)$ for some $Y_{3}^{\alpha}, Y_{1}^{\alpha}, Y_{0}^{\alpha} \notin\{\varnothing\}$, $\delta, \bar{\beta} \in B_{0}$. Then quasinormal representation of $\delta$ has a form

$$
\delta=\left(Y_{3}^{\delta} \times Z_{3}\right) \cup\left(Y_{2}^{\delta} \times Z_{2}\right) \cup\left(Y_{1}^{\delta} \times Z_{1}\right) \cup\left(Y_{0}^{\delta} \times \breve{D}\right)
$$

where $Y_{3}^{\delta}, Y_{1}^{\delta}, Y_{0}^{\delta} \notin\{\varnothing\}$. We suppose that

$$
\bar{\beta}=\left(P_{2} \times Z_{3}\right) \cup\left(P_{1} \times Z_{2}\right) \cup\left(P_{3} \times Z_{1}\right) \cup \bigcup_{t^{\prime} \in X \backslash \bar{D}}\left(\left\{t^{\prime}\right\} \times \bar{\beta}_{2}\left(t^{\prime}\right)\right)
$$

where $\bar{\beta}_{1}=\left(\begin{array}{llll}\varnothing & P_{1} & P_{2} & P_{3} \\ \varnothing & Z_{2} & Z_{3} & Z_{1}\end{array}\right)$ is normal mapping for $\bar{\beta}$ and $\bar{\beta}_{2}$ is complement mapping of the set $X \backslash \breve{D}$ on the set $\tilde{D}$. So, $\bar{\beta} \in B_{0}$ since $V\left(X^{*}, \bar{\beta}\right)=D$. From the equalities (2.1) and definition of $\bar{\beta}$

$$
\begin{aligned}
Z_{3} \bar{\beta} & =P_{2} \bar{\beta}=Z_{3} \\
Z_{2} \bar{\beta} & =\left(P_{1} \cup P_{3}\right) \bar{\beta}=P_{1} \bar{\beta} \cup P_{3} \bar{\beta}=Z_{2} \cup Z_{1}=\breve{D} \\
Z_{1} \bar{\beta} & =\left(P_{2} \cup P_{3}\right) \bar{\beta}=P_{2} \bar{\beta} \cup P_{3} \bar{\beta}=Z_{3} \cup Z_{1}=Z_{1} \\
\breve{D} \bar{\beta} & =\left(P_{1} \cup P_{2} \cup P_{3}\right) \bar{\beta}=P_{1} \bar{\beta} \cup P_{2} \bar{\beta} \cup P_{3} \bar{\beta}=Z_{2} \cup \breve{D} \cup Z_{1}=\breve{D} \\
\delta \circ \bar{\beta} & =\left(Y_{3}^{\delta} \times Z_{3} \bar{\beta}\right) \cup\left(Y_{2}^{\delta} \times Z_{2} \bar{\beta}\right) \cup\left(Y_{1}^{\delta} \times Z_{1} \bar{\beta}\right) \cup\left(Y_{0}^{\delta} \times \breve{D} \bar{\beta}\right) \\
& =\left(Y_{3}^{\delta} \times \breve{D}\right) \cup\left(Y_{2}^{\delta} \times \breve{D}\right) \cup\left(Y_{1}^{\delta} \times \breve{D}\right) \cup\left(Y_{0}^{\delta} \times \breve{D}\right) \\
& =\left(Y_{3}^{\delta} \times \breve{D}\right) \cup\left(Y_{1}^{\delta} \times \breve{D}\right) \cup\left(\left(Y_{2}^{\delta} \cup Y_{0}^{\delta}\right) \times \breve{D}\right)=\alpha .
\end{aligned}
$$

b. Let $\alpha \in B_{0} \circ \beta_{0} \cup \tilde{B}_{32}$. Then $\alpha \in B_{0} \circ \beta_{0}$ or $\alpha \in \tilde{B}_{32}$. If $\alpha \in B_{0} \circ \beta_{0}$ then $\alpha=\delta \circ \beta_{0}$ for some $\delta \in B_{0}$. In this case we have

$$
\delta=\left(Y_{3}^{\delta} \times Z_{3}\right) \cup\left(Y_{2}^{\delta} \times Z_{2}\right) \cup\left(Y_{1}^{\delta} \times Z_{1}\right) \cup\left(Y_{0}^{\delta} \times \breve{D}\right)
$$

where $Y_{3}^{\delta}, Y_{1}^{\delta}, Y_{0}^{\delta} \notin\{\varnothing\}$. Also

$$
\begin{aligned}
\alpha & =\delta \circ \beta_{0}=\left(Y_{3}^{\delta} \times Z_{3} \beta_{0}\right) \cup\left(Y_{2}^{\delta} \times Z_{2} \beta_{0}\right) \cup\left(Y_{1}^{\delta} \times Z_{1} \beta_{0}\right) \cup\left(Y_{0}^{\delta} \times \breve{D} \beta_{0}\right) \\
& =\left(Y_{3}^{\delta} \times Z_{3}\right) \cup\left(Y_{2}^{\delta} \times Z_{2}\right) \cup\left(Y_{1}^{\delta} \times Z_{1}\right) \cup\left(Y_{0}^{\delta} \times \breve{D}\right) \\
& =\left(Y_{3}^{\delta} \times Z_{3}\right) \cup\left(Y_{2}^{\delta} \times Z_{2}\right) \cup\left(\left(Y_{1}^{\delta} \cup Y_{0}^{\delta}\right) \times \breve{D}\right) \in B_{32} \backslash \tilde{B}_{32}
\end{aligned}
$$

is satisfied. So, we have $\left(B_{0} \circ \beta_{0}\right) \cup \tilde{B}_{32} \subseteq B_{32}$. On the other hand, if $\alpha \in \tilde{B}_{32} \subseteq B_{32}$ then $\left(B_{0} \circ \beta_{0}\right) \cup \tilde{B}_{32} \subseteq B_{32}$ is satisfied. Conversely, if $\alpha \in B_{32}$ then quasinormal representation of $\alpha$ has a form

$$
\alpha=\left(Y_{3}^{\alpha} \times Z_{3}\right) \cup\left(Y_{2}^{\alpha} \times Z_{2}\right) \cup\left(Y_{0}^{\alpha} \times \breve{D}\right)
$$

where $Y_{3}^{\alpha}, Y_{2}^{\alpha}, Y_{0}^{\alpha} \notin\{\varnothing\}$ or $Y_{3}^{\alpha}, Y_{2}^{\alpha} \notin\{\varnothing\}$ and $Y_{0}^{\alpha}=\varnothing$. We suppose that $Y_{3}^{\alpha}, Y_{2}^{\alpha} \notin\{\varnothing\}$. In this case, we have

$$
\begin{aligned}
\delta \circ \beta_{0} & =\left(Y_{3}^{\delta} \times Z_{3} \beta_{0}\right) \cup\left(Y_{2}^{\delta} \times Z_{2} \beta_{0}\right) \cup\left(Y_{0}^{\delta} \times Z_{1} \beta_{0}\right) \\
& =\left(Y_{3}^{\delta} \times Z_{3}\right) \cup\left(Y_{2}^{\delta} \times Z_{2}\right)\left(Y_{0}^{\delta} \times \breve{D}\right)=\alpha
\end{aligned}
$$

for $\delta=\left(Y_{3}^{\alpha} \times Z_{3}\right) \cup\left(Y_{2}^{\alpha} \times Z_{2}\right) \cup\left(Y_{0}^{\alpha} \times Z_{1}\right) \in B_{0}$. So, we have $B_{32} \subseteq\left(B_{0} \circ \beta_{0}\right) \cup \tilde{B}_{32}$. Now suppose that $Y_{3}^{\alpha}, Y_{2}^{\alpha} \notin\{\varnothing\}$ and $Y_{0}^{\alpha}=\varnothing$. In this case, we have 
$\alpha \in \tilde{B}_{32} \subseteq\left(B_{0} \circ \beta_{0}\right) \cup \tilde{B}_{32}$. So, $\left(B_{0} \circ \beta_{0}\right) \cup \tilde{B}_{32}=B_{32}$.

g. From the statement $c$, we have that $\left(B_{0} \circ \beta_{0}\right) \cup \tilde{B}_{32}=B_{32}$ where $\beta_{0} \in \tilde{B}_{32}$ by definition of $\beta_{0}$. Thus, every element of the set $B_{32}$ is product of elements of the set $B_{0} \cup \tilde{B}_{32}$.

Lemma 3. Let $D=\left\{Z_{3}, Z_{2}, Z_{1}, \breve{D}\right\} \in \Sigma_{2.2}(X, 4)$. If $|X \backslash \breve{D}| \geq 1$ then the following statements are true.

a) If $\alpha=X \times \breve{D}$ then $\alpha$ is product of elements of the set $B_{0}$.

b) If $\alpha=X \times Z_{1}$ then $\alpha$ is product of elements of the set $B_{0}$.

c) If $\alpha=\left(Y_{3}^{\alpha} \times Z_{3}\right) \cup\left(Y_{1}^{\alpha} \times Z_{1}\right)$ for some $Y_{3}^{\alpha}, Y_{1}^{\alpha} \notin \varnothing$, then $\alpha$ is product of elements of the $B_{0}$.

d) If $\alpha=\left(Y_{3}^{\alpha} \times Z_{3}\right) \cup\left(Y_{0}^{\alpha} \times \breve{D}\right)$ for some $Y_{3}^{\alpha}, Y_{0}^{\alpha} \notin \varnothing$, then $\alpha$ is product of elements of the $B_{0}$.

e) If $\alpha=\left(Y_{2}^{\alpha} \times Z_{2}\right) \cup\left(Y_{0}^{\alpha} \times \breve{D}\right)$ for some $Y_{2}^{\alpha}, Y_{0}^{\alpha} \notin \varnothing$, then $\alpha$ is product of elements of the $B_{0}$.

f) If $\alpha=\left(Y_{1}^{\alpha} \times Z_{1}\right) \cup\left(Y_{0}^{\alpha} \times \breve{D}\right)$ for some $Y_{1}^{\alpha}, Y_{0}^{\alpha} \notin \varnothing$, then $\alpha$ is product of elements of the $B_{0}$.

Proof. c. Let quasinormal representation of $\alpha$ has a form $\alpha=\left(Y_{3}^{\alpha} \times Z_{3}\right) \cup\left(Y_{1}^{\alpha} \times Z_{1}\right)$ where $Y_{3}^{\delta}, Y_{1}^{\delta} \notin\{\varnothing\}$. By definition of the semilattice $D,|X| \geq 3$. We suppose that $\left|Y_{3}^{\alpha}\right| \geq 1$ and $\left|Y_{1}^{\alpha}\right| \geq 2$. In this case, we suppose that

$$
\bar{\beta}=\left(P_{2} \times Z_{3}\right) \cup\left(\left(P_{1} \cup P_{3}\right) \times Z_{1}\right) \cup \bigcup_{t^{\prime} \in X \backslash \bar{D}}\left(\left\{t^{\prime}\right\} \times \bar{\beta}_{2}\left(t^{\prime}\right)\right)
$$

where $\bar{\beta}_{1}=\left(\begin{array}{llll}\varnothing & P_{1} & P_{2} & P_{3} \\ \varnothing & Z_{1} & Z_{3} & Z_{1}\end{array}\right)$ is normal mapping for $\bar{\beta}$ and $\bar{\beta}_{2}$ is complement mapping of the set $X \times \breve{D}$ on the set $\tilde{D} \backslash\left\{Z_{3}, Z_{1}\right\}=\left\{Z_{2}\right\}$ (by suppose $|X \backslash \breve{D}| \geq 1 \quad$ ). So, $\bar{\beta} \in B_{0} \quad$ since $V\left(X^{*}, \bar{\beta}\right)=D$. Also, $Y_{3}^{\delta}=Y_{3}^{\alpha} \quad$ and $Y_{2}^{\delta} \cup Y_{1}^{\delta} \cup Y_{0}^{\delta}=Y_{1}^{\delta}$ since $\left|Y_{3}^{\delta}\right| \geq 1,\left|Y_{2}^{\delta}\right| \geq 1,\left|Y_{1}^{\delta}\right| \geq 1,\left|Y_{0}^{\delta}\right| \geq 0$. From the equalities (2.1) and definition of $\bar{\beta}$ we obtain that

$$
\begin{aligned}
Z_{3} \bar{\beta}= & P_{2} \bar{\beta}=Z_{3} \\
Z_{2} \bar{\beta}= & \left(P_{1} \cup P_{3}\right) \bar{\beta}=P_{1} \bar{\beta} \cup P_{3} \bar{\beta}=Z_{1} \cup Z_{1}=Z_{1} \\
Z_{1} \bar{\beta}= & \left(P_{2} \cup P_{3}\right) \bar{\beta}=P_{2} \bar{\beta} \cup P_{3} \bar{\beta}=Z_{3} \cup Z_{1}=Z_{1} \\
\bar{D} \bar{\beta}= & \left(P_{1} \cup P_{2} \cup P_{3}\right) \bar{\beta}=P_{1} \bar{\beta} \cup P_{2} \bar{\beta} \cup P_{3} \bar{\beta}=Z_{1} \cup Z_{3} \cup Z_{1}=Z_{1} \\
\delta \circ \bar{\beta} & =\left(Y_{3}^{\delta} \times Z_{3} \bar{\beta}\right) \cup\left(Y_{2}^{\delta} \times Z_{2} \bar{\beta}\right) \cup\left(Y_{1}^{\delta} \times Z_{1} \bar{\beta}\right) \cup\left(Y_{0}^{\delta} \times \bar{D} \bar{\beta}\right) \\
& =\left(Y_{3}^{\delta} \times Z_{3}\right) \cup\left(Y_{2}^{\delta} \times Z_{1}\right) \cup\left(Y_{1}^{\delta} \times Z_{1}\right) \cup\left(Y_{0}^{\delta} \times Z_{1}\right) \\
& =\left(Y_{3}^{\delta} \times Z_{3}\right) \cup\left(\left(Y_{2}^{\delta} \cup Y_{1}^{\delta} \cup Y_{0}^{\delta}\right) \times Z_{1}\right)=\alpha
\end{aligned}
$$

Now, we suppose that $\left|Y_{3}^{\alpha}\right| \geq 2$ and $\left|Y_{1}^{\alpha}\right| \geq 1$. In this case, we suppose that

$$
\bar{\beta}=\left(\left(P_{2} \cup P_{3}\right) \times Z_{3}\right) \cup\left(P_{1} \times Z_{1}\right) \cup \bigcup_{t^{\prime} \in X \backslash \bar{D}}\left(\left\{t^{\prime}\right\} \times \bar{\beta}_{2}\left(t^{\prime}\right)\right)
$$

where $\bar{\beta}_{1}=\left(\begin{array}{llll}\varnothing & P_{1} & P_{2} & P_{3} \\ \varnothing & Z_{1} & Z_{3} & Z_{3}\end{array}\right)$ is normal mapping for $\bar{\beta}$ and $\bar{\beta}_{2}$ is complement mapping of the set $X \times \breve{D}$ on the set $\tilde{D} \backslash\left\{Z_{3}, Z_{1}\right\}=\left\{Z_{2}\right\}$ (by suppose 
$|X \backslash \breve{D}| \geq 1$ ). So, $\bar{\beta} \in B_{0}$ since $V\left(X^{*}, \bar{\beta}\right)=D$. Also, $Y_{3}^{\delta} \cup Y_{1}^{\delta}=Y_{3}^{\alpha} \quad$ and $Y_{2}^{\delta} \cup Y_{0}^{\delta}=Y_{1}^{\alpha}$ since $\left|Y_{3}^{\delta}\right| \geq 1,\left|Y_{2}^{\delta}\right| \geq 1,\left|Y_{1}^{\delta}\right| \geq 1,\left|Y_{0}^{\delta}\right| \geq 0$. From the equalities (2.1) and definition of $\bar{\beta}$ we obtain that

$$
\begin{aligned}
Z_{3} \bar{\beta}= & P_{2} \bar{\beta}=Z_{3} \\
Z_{2} \bar{\beta}= & \left(P_{1} \cup P_{3}\right) \bar{\beta}=P_{1} \bar{\beta} \cup P_{3} \bar{\beta}=Z_{1} \cup Z_{3}=Z_{1} \\
Z_{1} \bar{\beta}= & \left(P_{2} \cup P_{3}\right) \bar{\beta}=P_{2} \bar{\beta} \cup P_{3} \bar{\beta}=Z_{3} \cup Z_{3}=Z_{3} \\
\bar{D} \bar{\beta}= & \left(P_{1} \cup P_{2} \cup P_{3}\right) \bar{\beta}=P_{1} \bar{\beta} \cup P_{2} \bar{\beta} \cup P_{3} \bar{\beta}=Z_{1} \cup Z_{3} \cup Z_{3}=Z_{1} \\
\delta \circ \bar{\beta} & =\left(Y_{3}^{\delta} \times Z_{3} \bar{\beta}\right) \cup\left(Y_{2}^{\delta} \times Z_{2} \bar{\beta}\right) \cup\left(Y_{1}^{\delta} \times Z_{1} \bar{\beta}\right) \cup\left(Y_{0}^{\delta} \times \bar{D} \bar{\beta}\right) \\
& =\left(Y_{3}^{\delta} \times Z_{3}\right) \cup\left(Y_{2}^{\delta} \times Z_{1}\right) \cup\left(Y_{1}^{\delta} \times Z_{3}\right) \cup\left(Y_{0}^{\delta} \times Z_{1}\right) \\
& =\left(\left(Y_{3}^{\delta} \cup Y_{1}^{\delta}\right) \times Z_{3}\right) \cup\left(\left(Y_{2}^{\delta} \cup Y_{0}^{\delta}\right) \times Z_{1}\right)=\alpha
\end{aligned}
$$

Lemma 4. Let $D=\left\{Z_{3}, Z_{2}, Z_{1}, \breve{D}\right\} \in \Sigma_{2.2}(X, 4)$,

$\sigma_{0}=\left(Z_{3} \times Z_{3}\right) \cup\left(\left(X \backslash Z_{3}\right) \times Z_{1}\right)$ and $\sigma_{1}=\left(Z_{2} \times Z_{2}\right) \cup\left(\left(X \backslash Z_{2}\right) \times Z_{1}\right)$. If $X=\breve{D}$ then the following statements are true

a) If $\alpha=\left(Y_{3}^{\alpha} \times Z_{3}\right) \cup\left(Y_{0}^{\alpha} \times \breve{D}\right)$ for some $Y_{3}^{\alpha}, Y_{0}^{\alpha} \notin\{\varnothing\}$, then $\alpha$ is product of elements of the $B_{0} \cup B_{32}$.

b) If $\alpha=\left(Y_{2}^{\alpha} \times Z_{2}\right) \cup\left(Y_{0}^{\alpha} \times \breve{D}\right)$ for some $Y_{2}^{\alpha}, Y_{0}^{\alpha} \notin\{\varnothing\}$, then $\alpha$ is product of elements of the $B_{32} \cup\left\{\sigma_{1}\right\}$.

c) If $\alpha=\left(Y_{1}^{\alpha} \times Z_{1}\right) \cup\left(Y_{0}^{\alpha} \times \breve{D}\right)$ for some $Y_{1}^{\alpha}, Y_{0}^{\alpha} \notin\{\varnothing\}$, then $\alpha$ is product of elements of the $B_{32} \cup\left\{\sigma_{0}, \sigma_{1}\right\}$.

Proof. First, remark that $Z_{3} \sigma_{0}=Z_{3}, Z_{2} \sigma_{0}=\breve{D} \sigma_{0}=Z_{1}, Z_{3} \sigma_{1}=Z_{1}, Z_{2} \sigma_{1}=Z_{2}$, $\breve{D} \sigma_{1}=\breve{D}$.

a. Let $\alpha=\left(Y_{3}^{\alpha} \times Z_{3}\right) \cup\left(Y_{0}^{\alpha} \times \breve{D}\right)$ for some $Y_{3}^{\alpha}, Y_{0}^{\alpha} \notin \varnothing$. In this case, we suppose that

$$
\delta=\left(Y_{3}^{\delta} \times Z_{3}\right) \cup\left(Y_{2}^{\delta} \times Z_{2}\right) \cup\left(Y_{0}^{\delta} \times \breve{D}\right)
$$

and

$$
\beta_{1}=\left(Z_{3} \times Z_{3}\right) \cup\left(\left(Z_{1} \backslash Z_{3}\right) \times Z_{1}\right) \cup\left(\left(X \backslash Z_{1}\right) \times \breve{D}\right)
$$

where $Y_{3}^{\delta}, Y_{2}^{\delta} \notin\{\varnothing\}$. It is easy to see that $\delta \in B_{32}$ and $\beta_{1}$ is generating by elements of the $B_{0}$ by statement $\mathrm{b}$ of Lemma 2. Also, $Y_{3}^{\delta}=Y_{3}^{\alpha}$ and $Y_{2}^{\delta} \cup Y_{0}^{\delta}=Y_{0}^{\alpha} \quad$ since $Z_{3} \bar{\beta}=Z_{3}, \quad Z_{2} \bar{\beta}=\breve{D} \bar{\beta}=\breve{D}$ and $\left|Y_{3}^{\delta}\right| \geq 1,\left|Y_{2}^{\delta}\right| \geq 1,\left|Y_{0}^{\delta}\right| \geq 0$. So, $\alpha$ is product of elements of the $B_{0} \cup B_{32}$.

Lemma 5. Let

$$
D=\left\{Z_{3}, Z_{2}, Z_{1}, \breve{D}\right\} \in \Sigma_{2.2}(X, 4)
$$

and

$$
\sigma_{1}=\left(Z_{2} \times Z_{2}\right) \cup\left(\left(X \backslash Z_{2}\right) \times Z_{1}\right) .
$$

If $|X \backslash \breve{D}| \geq 1$ then $S_{1}=B_{0} \cup \tilde{B}_{32} \cup\left\{\sigma_{1}\right\}$ is an irreducible generating set for the semigroup $B_{X}(D)$.

Proof. First, we must prove that every element of $B_{X}(D)$ is product of ele- 
ments of $S_{1}$. Let $\alpha \in B_{X}(D)$ and

$$
\alpha=\left(Y_{3}^{\alpha} \times Z_{3}\right) \cup\left(Y_{2}^{\alpha} \times Z_{2}\right) \cup\left(Y_{1}^{\alpha} \times Z_{1}\right) \cup\left(Y_{0}^{\alpha} \times \breve{D}\right)
$$

where $Y_{3}^{\alpha} \cup Y_{2}^{\alpha} \cup Y_{1}^{\alpha} \cup Y_{0}^{\alpha}=X$ and $Y_{3}^{\alpha} \cap Y_{2}^{\alpha}=\varnothing, \quad(0 \leq i \neq j \leq 3)$. We suppose that $\left|V\left(X^{*}, \alpha\right)\right|=1$. Then we have $V\left(X^{*}, \alpha\right) \in\left\{\left\{Z_{3}\right\},\left\{Z_{2}\right\},\left\{Z_{1}\right\},\{\breve{D}\}\right\}$. If $V\left(X^{*}, \alpha\right) \in\left\{\left\{Z_{3}\right\},\left\{Z_{2}\right\},\left\{Z_{1}\right\}\right\}$ then $\alpha=X \times Z_{3}$ or $\alpha=X \times Z_{2}$ or $\alpha=X \times Z_{1}$. Quasinormal representations of $\delta, \beta_{1}, \beta_{2}$ and $\beta_{3}$ has form

$$
\begin{aligned}
& \delta=\left(Y_{3}^{\delta} \times Z_{3}\right) \cup\left(Y_{2}^{\delta} \times Z_{2}\right) \cup\left(Y_{1}^{\delta} \times Z_{1}\right) \cup\left(Y_{0}^{\delta} \times \breve{D}\right) \\
& \beta_{1}=\left(\breve{D} \times Z_{3}\right) \cup\left((X \backslash \breve{D}) \times Z_{2}\right) \\
& \beta_{2}=\left(\breve{D} \times Z_{2}\right) \cup\left((X \backslash \breve{D}) \times Z_{1}\right) \\
& \beta_{3}=\left(\breve{D} \times Z_{1}\right) \cup\left((X \backslash \breve{D}) \times Z_{2}\right)
\end{aligned}
$$

where $Y_{3}^{\delta}, Y_{2}^{\delta}, Y_{1}^{\delta} \notin\{\varnothing\}$. So, $\delta \in B_{0}, \quad \beta_{1} \in \tilde{B}_{32} \quad$ and $\beta_{2}, \beta_{3} \in B_{21}$ since $|X \backslash \breve{D}| \geq 1$. From the definition of $\delta, \beta_{1}, \beta_{2}$ and $\beta_{3}$ we obtain that

$$
\begin{aligned}
\delta \circ \beta_{1} & =\left(Y_{3}^{\delta} \times Z_{3} \beta_{1}\right) \cup\left(Y_{2}^{\delta} \times Z_{2} \beta_{1}\right) \cup\left(Y_{1}^{\delta} \times Z_{1} \beta_{1}\right) \cup\left(Y_{0}^{\delta} \times \breve{D} \beta_{1}\right) \\
& =\left(Y_{3}^{\delta} \times Z_{3}\right) \cup\left(Y_{2}^{\delta} \times Z_{3}\right) \cup\left(Y_{1}^{\delta} \times Z_{3}\right) \cup\left(Y_{0}^{\delta} \times Z_{3}\right) \\
& =\left(Y_{3}^{\delta} \cup Y_{2}^{\delta} \cup Y_{1}^{\delta} \cup Y_{0}^{\delta}\right) \times Z_{3}=X \times Z_{3} \\
\delta \circ \beta_{2} & =\left(Y_{3}^{\delta} \times Z_{3} \beta_{2}\right) \cup\left(Y_{2}^{\delta} \times Z_{2} \beta_{2}\right) \cup\left(Y_{1}^{\delta} \times Z_{1} \beta_{2}\right) \cup\left(Y_{0}^{\delta} \times \breve{D} \beta_{2}\right) \\
& =\left(Y_{3}^{\delta} \times Z_{2}\right) \cup\left(Y_{2}^{\delta} \times Z_{2}\right) \cup\left(Y_{1}^{\delta} \times Z_{2}\right) \cup\left(Y_{0}^{\delta} \times Z_{2}\right) \\
& =\left(Y_{3}^{\delta} \cup Y_{2}^{\delta} \cup Y_{1}^{\delta} \cup Y_{0}^{\delta}\right) \times Z_{2}=X \times Z_{2} \\
\delta \circ \beta_{3} & =\left(Y_{3}^{\delta} \times Z_{3} \beta_{3}\right) \cup\left(Y_{2}^{\delta} \times Z_{2} \beta_{3}\right) \cup\left(Y_{1}^{\delta} \times Z_{1} \beta_{3}\right) \cup\left(Y_{0}^{\delta} \times \breve{D} \beta_{3}\right) \\
& =\left(Y_{3}^{\delta} \times Z_{1}\right) \cup\left(Y_{2}^{\delta} \times Z_{1}\right) \cup\left(Y_{1}^{\delta} \times Z_{1}\right) \cup\left(Y_{0}^{\delta} \times Z_{1}\right) \\
& =\left(Y_{3}^{\delta} \cup Y_{2}^{\delta} \cup Y_{1}^{\delta} \cup Y_{0}^{\delta}\right) \times Z_{1}=X \times Z_{1}
\end{aligned}
$$

That means, $X \times Z_{1}, X \times Z_{2}$ and $X \times Z_{3}$ are generated by $B_{0} \cup \tilde{B}_{32}$, $B_{0} \cup B_{21}$ and $B_{0} \cup B_{21}$ respectively. By using statement $g$ and h of Lemma 3, we have $X \times Z_{1}, X \times Z_{2}$ and $X \times Z_{3}$ are generated by $B_{0} \cup \tilde{B}_{32} \cup\left\{\sigma_{1}\right\}$. On the other hand, if $V\left(X^{*}, \alpha\right)=\{\breve{D}\}$ then $\alpha=X \times \breve{D}$ By using statement a of Lemma 3, we have $\alpha$ is product of some elemets of $B_{0}$.

So, $S_{1}$ is generating set for the semigroup $B_{X}(D)$. Now, we must prove that $S_{1}=B_{0} \cup \tilde{B}_{32} \cup\left\{\sigma_{1}\right\}$ is irreducible. Let $\alpha \in S_{1}$.

If $\alpha \in B_{0}$ then $\alpha \neq \sigma \circ \tau$ for all $\sigma, \tau \in B_{X}(D) \backslash\{\alpha\}$ from Lemma 2. So, $\alpha \neq \sigma \circ \tau$ for all $\sigma, \tau \in S_{1} \backslash\{\alpha\}$. That means, $\alpha \notin B_{0}$.

If $\alpha \in \tilde{B}_{32}$ then the quasinormal representation of $\alpha$ has form $\alpha=\left(Y_{3}^{\alpha} \times Z_{3}\right) \cup\left(Y_{2}^{\alpha} \times Z_{2}\right)$ for some $Y_{3}^{\alpha}, Y_{2}^{\alpha} \notin \varnothing$. Let $\alpha=\delta \circ \beta$ for some $\delta, \beta \in S_{1} \backslash\{\alpha\}$.

We suppose that $\delta \in B_{0} \backslash\{\alpha\}$ and $\beta \in S_{1} \backslash\{\alpha\}$. By definition of $B_{0}$, quasinormal representation of $\delta$ has form

$$
\delta=\left(Y_{3}^{\delta} \times Z_{3}\right) \cup\left(Y_{2}^{\delta} \times Z_{2}\right) \cup\left(Y_{1}^{\delta} \times Z_{1}\right) \cup\left(Y_{0}^{\delta} \times \breve{D}\right)
$$


where $Y_{3}^{\delta}, Y_{2}^{\delta}, Y_{1}^{\delta} \notin\{\varnothing\}$. By using $Z_{3} \subset Z_{1} \subset \breve{D}$ and $Z_{2} \subset \breve{D}$ we have $Z_{3} \beta$ and $Z_{2} \beta$ are minimal elements of the semilattice $\left\{Z_{3} \beta, Z_{2} \beta, Z_{1} \beta, \breve{D} \beta\right\}$. Also, we have

$$
\begin{aligned}
& \left(Y_{3}^{\alpha} \times Z_{3}\right) \cup\left(Y_{2}^{\alpha} \times Z_{2}\right)=\alpha=\delta \circ \beta \\
& =\left(Y_{3}^{\delta} \times Z_{3} \beta\right) \cup\left(Y_{2}^{\delta} \times Z_{2} \beta\right) \cup\left(Y_{1}^{\delta} \times Z_{1} \beta\right) \cup\left(Y_{0}^{\delta} \times \breve{D} \beta\right)
\end{aligned}
$$

Since $Z_{3}$ and $Z_{2}$ are minimal elements of the semilattice $\left\{Z_{3}, Z_{2}, \breve{D}\right\}$, this equality is possible only if $Z_{3}=Z_{3} \beta, Z_{2}=Z_{2} \beta$ or $Z_{3}=Z_{2} \beta, Z_{2}=Z_{3} \beta$. By using formal equalities and $P_{3} \beta, P_{2} \beta, P_{1} \beta \in D$, we obtain

$$
\begin{array}{lll}
Z_{3}=Z_{3} \beta=P_{2} \beta & \text { and } & Z_{2}=Z_{2} \beta=P_{1} \beta=P_{3} \beta \\
Z_{2}=Z_{3} \beta=P_{2} \beta & \text { and } & Z_{3}=Z_{2} \beta=P_{1} \beta=P_{3} \beta
\end{array}
$$

respectively. Let $Z_{3}=P_{2} \beta$ and $Z_{2}=P_{1} \beta=P_{3} \beta$. If $\bar{\beta}$ is sub-quasinormal representation of $\beta$ then $\delta \circ \beta=\delta \circ \bar{\beta}$ and

$$
\bar{\beta}=\left(\left(P_{1} \cup P_{3}\right) \times Z_{2}\right) \cup\left(P_{2} \times Z_{3}\right) \cup \bigcup_{t^{\prime} \in X \backslash \bar{D}}\left(\left\{t^{\prime}\right\} \times \bar{\beta}_{2}\left(t^{\prime}\right)\right)
$$

where $\bar{\beta}_{1}=\left(\begin{array}{llll}\varnothing & P_{1} & P_{2} & P_{3} \\ \varnothing & Z_{2} & Z_{3} & Z_{2}\end{array}\right)$ is normal mapping for $\bar{\beta}$ and $\bar{\beta}_{2}$ is complement mapping of the set $X \times \breve{D}$ on the set $\tilde{D}=\left\{Z_{3}, Z_{2}, Z_{1}\right\}$. From formal equalities, we obtain

$$
\bar{\beta}=\left(Z_{2} \times Z_{2}\right) \cup\left(Z_{3} \times Z_{3}\right) \cup \bigcup_{t^{\prime} \in X \backslash \bar{D}}\left(\left\{t^{\prime}\right\} \times \bar{\beta}_{2}\left(t^{\prime}\right)\right) \in S_{1} \backslash\{\alpha\}
$$

and by using $Z_{1} \cap Z_{2} \neq \varnothing, Z_{3} \cup Z_{2}=D$ and $\left|Y_{1}^{\delta} \cup Y_{0}^{\delta}\right| \geq 1$, we have

$$
\begin{aligned}
\delta \circ \bar{\beta} & =\left(Y_{3}^{\delta} \times Z_{3} \bar{\beta}\right) \cup\left(Y_{2}^{\delta} \times Z_{2} \bar{\beta}\right) \cup\left(Y_{1}^{\delta} \times Z_{1} \bar{\beta}\right) \cup\left(Y_{0}^{\delta} \times \breve{D} \bar{\beta}\right) \\
& =\left(Y_{3}^{\delta} \times Z_{3}\right) \cup\left(Y_{2}^{\delta} \times Z_{2}\right) \cup\left(Y_{1}^{\delta} \times \breve{D}\right) \cup\left(Y_{0}^{\delta} \times \breve{D}\right) \\
& =\left(Y_{3}^{\delta} \times Z_{3}\right) \cup\left(Y_{2}^{\delta} \times Z_{2}\right) \cup\left(\left(Y_{1}^{\delta} \cup Y_{0}^{\delta}\right) \times \breve{D}\right) \neq \alpha
\end{aligned}
$$

This contradicts with $\alpha=\delta \circ \beta$. So, $\delta \notin B_{0} \backslash\{\alpha\}$.

Now, we suppose that $\delta \in \tilde{B}_{32} \backslash\{\alpha\}$ and $\beta \in S_{1} \backslash\{\alpha\}$. Similar operations are applied as above, we obtain $\delta \notin \tilde{B}_{32} \backslash\{\alpha\}$.

Now, we suppose that $\delta=\sigma_{1}$ and $\beta \in S_{1} \backslash\{\alpha\}$. Similar operations are applied as above, we obtain $\delta \neq \sigma_{1}$.

That means $\alpha \neq \delta \circ \beta$ for any $\alpha \in \tilde{B}_{32}$ and $\delta, \beta \in S_{1} \backslash\{\alpha\}$.

If $\alpha=\sigma_{1}$, then by the definition of $\sigma_{1}$, quasinormal representation of $\alpha$ has a form $\alpha=\left(Z_{2} \times Z_{2}\right) \cup\left(\left(X \backslash Z_{2}\right) \times Z_{1}\right)$. Let $\alpha=\delta \circ \beta$ for some $\delta, \beta \in S_{1} \backslash\left\{\sigma_{1}\right\}$.

We suppose that $\delta \in B_{0} \backslash\left\{\sigma_{1}\right\}$ and $\beta \in S_{1} \backslash\left\{\sigma_{1}\right\}$. By definition of $B_{0}$, quasinormal representation of $\delta$ has form

$$
\delta=\left(Y_{3}^{\delta} \times Z_{3}\right) \cup\left(Y_{2}^{\delta} \times Z_{2}\right) \cup\left(Y_{1}^{\delta} \times Z_{1}\right) \cup\left(Y_{0}^{\delta} \times \breve{D}\right)
$$

where $Y_{3}^{\delta}, Y_{2}^{\delta}, Y_{1}^{\delta} \notin\{\varnothing\}$. By using $Z_{3} \subset Z_{1} \subset \breve{D}$ and $Z_{2} \subset \breve{D}$ we have $Z_{3} \beta$ and $Z_{2} \beta$ are minimal elements of the semilattice $\left\{Z_{3} \beta, Z_{2} \beta, Z_{1} \beta, \breve{D} \beta\right\}$. Also, 
we have

$$
\begin{aligned}
& \left(Z_{2} \times Z_{2}\right) \cup\left(\left(X \backslash Z_{2}\right) \times Z_{1}\right)=\alpha=\delta \circ \beta \\
& =\left(Y_{3}^{\delta} \times Z_{3} \beta\right) \cup\left(Y_{2}^{\delta} \times Z_{2} \beta\right) \cup\left(Y_{1}^{\delta} \times Z_{1} \beta\right) \cup\left(Y_{0}^{\delta} \times \breve{D} \beta\right)
\end{aligned}
$$

From $Z_{2}$ and $Z_{1}$ are minimal elements of the semilattice $\left\{Z_{2}, Z_{1}, \breve{D}\right\}$, this equality is possible only if $Z_{2}=Z_{3} \beta, Z_{1}=Z_{2} \beta$ or $Z_{2}=Z_{2} \beta, Z_{1}=Z_{3} \beta$. By using formal equalities, we obtain

$$
\begin{array}{lll}
Z_{2}=Z_{3} \beta=P_{2} \beta & \text { and } & Z_{1}=Z_{2} \beta=P_{1} \beta \bigcup P_{3} \beta \\
Z_{1}=Z_{3} \beta=P_{2} \beta & \text { and } & Z_{2}=Z_{2} \beta=P_{1} \beta=P_{3} \beta
\end{array}
$$

respectively. Let $Z_{2}=P_{2} \beta$ and $Z_{1}=P_{1} \beta \cup P_{3} \beta$ where $P_{1} \beta, P_{3} \beta \in\left\{Z_{3}, Z_{1}\right\}$. Then subquasinormal representation of $\beta$ has one of the form

$$
\begin{aligned}
& \bar{\beta}^{1}=\left(P_{1} \times Z_{3}\right) \cup\left(P_{2} \times Z_{2}\right) \cup\left(P_{3} \times Z_{1}\right) \cup \bigcup_{t^{\prime} \in X \backslash \breve{D}}\left(\left\{t^{\prime}\right\} \times \bar{\beta}_{2}\left(t^{\prime}\right)\right) \\
& \bar{\beta}^{2}=\left(P_{3} \times Z_{3}\right) \cup\left(P_{2} \times Z_{2}\right) \cup\left(P_{1} \times Z_{1}\right) \cup \bigcup_{t^{\prime} \in X \backslash \bar{D}}\left(\left\{t^{\prime}\right\} \times \bar{\beta}_{2}\left(t^{\prime}\right)\right) \\
& \bar{\beta}^{3}=\left(P_{2} \times Z_{2}\right) \cup\left(\left(P_{1} \cup P_{3}\right) \times Z_{1}\right) \cup \bigcup_{t^{\prime} \in X \backslash \bar{D}}\left(\left\{t^{\prime}\right\} \times \bar{\beta}_{2}\left(t^{\prime}\right)\right)
\end{aligned}
$$

where

$$
\bar{\beta}_{1}^{1}=\left(\begin{array}{llll}
\varnothing & P_{1} & P_{2} & P_{3} \\
\varnothing & Z_{3} & Z_{2} & Z_{1}
\end{array}\right), \quad \bar{\beta}_{1}^{2}=\left(\begin{array}{llll}
\varnothing & P_{1} & P_{2} & P_{3} \\
\varnothing & Z_{1} & Z_{2} & Z_{3}
\end{array}\right), \quad \bar{\beta}_{1}^{3}=\left(\begin{array}{llll}
\varnothing & P_{1} & P_{2} & P_{3} \\
\varnothing & Z_{1} & Z_{2} & Z_{1}
\end{array}\right)
$$

are normal mapping for $\bar{\beta}, \bar{\beta}_{2}$ is complement mapping of the set $X \times \breve{D}$ on the set $\tilde{D}=\left\{Z_{3}, Z_{2}, Z_{1}\right\}$ and $\delta \circ \beta=\delta \circ \bar{\beta}_{i}$. From formal equalities, we obtain

$$
\begin{aligned}
& \bar{\beta}^{1}=\left(\left(Z_{2} \backslash Z_{1}\right) \times Z_{3}\right) \cup\left(\left(Z_{1} \backslash Z_{2}\right) \times Z_{2}\right) \cup\left(\left(Z_{2} \backslash Z_{1}\right) \times Z_{1}\right) \cup \bigcup_{t^{\prime} \in X \backslash \breve{D}}\left(\left\{t^{\prime}\right\} \times \bar{\beta}_{2}\left(t^{\prime}\right)\right) \\
& \bar{\beta}^{2}=\left(\left(Z_{2} \cap Z_{1}\right) \times Z_{3}\right) \cup\left(\left(Z_{1} \backslash Z_{2}\right) \times Z_{2}\right) \cup\left(\left(Z_{2} \backslash Z_{1}\right) \times Z_{1}\right) \cup \bigcup_{t^{\prime} \in X \backslash \breve{D}}\left(\left\{t^{\prime}\right\} \times \bar{\beta}_{2}\left(t^{\prime}\right)\right) \\
& \bar{\beta}^{3}=\left(\left(Z_{1} \backslash Z_{2}\right) \times Z_{2}\right) \cup\left(Z_{2} \times Z_{1}\right) \cup \bigcup_{t^{\prime} \in X \backslash \bar{D}}\left(\left\{t^{\prime}\right\} \times \bar{\beta}_{2}\left(t^{\prime}\right)\right)
\end{aligned}
$$

and by using $\left|Y_{1}^{\delta} \cup Y_{0}^{\delta}\right| \geq 1$, we have

$$
\begin{aligned}
\delta \circ \bar{\beta}^{1} & =\delta \circ \bar{\beta}^{2}=\delta \circ \bar{\beta}^{3} \\
& =\left(Y_{3}^{\delta} \times Z_{3} \bar{\beta}^{1}\right) \cup\left(Y_{2}^{\delta} \times Z_{2} \bar{\beta}^{1}\right) \cup\left(Y_{1}^{\delta} \times Z_{1} \bar{\beta}^{1}\right) \cup\left(Y_{0}^{\delta} \times \breve{D} \bar{\beta}^{1}\right) \\
& =\left(Y_{3}^{\delta} \times Z_{2}\right) \cup\left(Y_{2}^{\delta} \times Z_{1}\right) \cup\left(Y_{1}^{\delta} \times \breve{D}\right) \cup\left(Y_{0}^{\delta} \times \breve{D}\right) \\
& =\left(Y_{3}^{\delta} \times Z_{2}\right) \cup\left(Y_{2}^{\delta} \times Z_{1}\right) \cup\left(\left(Y_{1}^{\delta} \cup Y_{0}^{\delta}\right) \times \breve{D}\right) \neq \alpha
\end{aligned}
$$

This contradicts with $\alpha=\delta \circ \beta$. So, $\delta \notin B_{0} \backslash\left\{\sigma_{1}\right\}$.

Now, we suppose that $\delta \in \tilde{B}_{32} \backslash\left\{\sigma_{1}\right\}$ and $\beta \in S_{1} \backslash\left\{\sigma_{1}\right\}$. Similar operations are applied as above, we obtain $\delta \notin \tilde{B}_{32} \backslash\left\{\sigma_{1}\right\}$.

That means $\alpha \neq \delta \circ \beta$ for any $\alpha \in \tilde{B}_{32}$ and $\delta, \beta \in S_{1} \backslash\{\alpha\}$.

Lemma 6. Let $D=\left\{Z_{3}, Z_{2}, Z_{1}, \breve{D}\right\} \in \Sigma_{2.2}(X, 4), \sigma_{0}=\left(Z_{3} \times Z_{3}\right) \cup\left(\left(X \backslash Z_{3}\right) \times Z_{1}\right)$ and $\sigma_{1}=\left(Z_{2} \times Z_{2}\right) \cup\left(\left(X \backslash Z_{2}\right) \times Z_{1}\right)$. If $X=\breve{D}$ then $S_{2}=B_{0} \cup \tilde{B}_{32} \cup\left\{\sigma_{0}, \sigma_{1}\right\}$ is irreducible generating set for the semigroup $B_{X}(D)$. 
Theorem 7. Let $D=\left\{Z_{3}, Z_{2}, Z_{1}, \breve{D}\right\} \in \Sigma_{2.2}(X, 4)$, $\sigma_{0}=\left(Z_{3} \times Z_{3}\right) \cup\left(\left(X \backslash Z_{3}\right) \times Z_{1}\right)$ and $\sigma_{1}=\left(Z_{2} \times Z_{2}\right) \cup\left(\left(X \backslash Z_{2}\right) \times Z_{1}\right)$. If $X$ is a finite set and $|X|=n$ then the following statements are true

a) If $|X \backslash \breve{D}| \geq 1$ then $\left|B_{0} \cup \tilde{B}_{32} \cup\left\{\sigma_{1}\right\}\right|=4^{n}-3^{n+1}+2^{n+2}-2$

b) If $X=\breve{D}$ then $\left|B_{0} \cup \tilde{B}_{32} \cup\left\{\sigma_{0}, \sigma_{1}\right\}\right|=4^{n}-3^{n+1}+2^{n+2}-1$

\section{Proof. Let}

$$
S_{n}=\left\{\varphi_{i} \mid \varphi_{i}: M=\{1,2, \cdots, n\} \rightarrow M=\{1,2, \cdots, n\} \text {, one to one mapping }\right\}
$$

be a group, $\varphi_{i_{1}}, \varphi_{i_{2}}, \cdots, \varphi_{i_{m}} \in S_{n}(m \leq n)$ and $Y_{\varphi_{1}}, Y_{\varphi_{2}}, \cdots, Y_{\varphi_{m}}$ be partitioning of $X$. It is well known that $k_{n}^{m}=\left|\left\{Y_{\varphi_{1}}, Y_{\varphi_{2}}, \cdots, Y_{\varphi_{m}}\right\}\right|=\sum_{i=1}^{m} \frac{(-1)^{m+i}}{(i-1) !(m-i) !}$. If $m=2,3,4$ then we have

$$
\begin{aligned}
& k_{n}^{2}=2^{n-1}-1 \\
& k_{n}^{3}=\frac{1}{2} \cdot 3^{n-1}-2^{n-1}+\frac{1}{2} \\
& k_{n}^{4}=\frac{1}{6} \cdot 4^{n-1}-\frac{1}{2} \cdot 3^{n-1}+\frac{1}{2} \cdot 2^{n-1}-\frac{1}{6}
\end{aligned}
$$

If $Y_{\varphi_{1}}, Y_{\varphi_{2}}$ are any two elements of partitioning of $X$ and $\bar{\beta}=\left(Y_{\varphi_{1}} \times T_{1}\right) \cup\left(Y_{\varphi_{2}} \times T_{2}\right)$ where $T_{1}, T_{2} \in D$ and $T_{1} \neq T_{2}$, then the number of different binary relations $\bar{\beta}$ of semigroup $B_{X}(D)$ is equal to

$$
2 \cdot k_{n}^{2}=2^{n}-2
$$

If $Y_{\varphi_{1}}, Y_{\varphi_{2}}, Y_{\varphi_{3}}$ are any three elements of partitioning of $X$ and $\bar{\beta}=\left(Y_{\varphi_{1}} \times T_{1}\right) \cup\left(Y_{\varphi_{2}} \times T_{2}\right) \cup\left(Y_{\varphi_{3}} \times T_{3}\right)$ where $T_{1}, T_{2}, T_{3}$ are pairwise different elements of $D$, then the number of different binary relations $\bar{\beta}$ of semigroup $B_{X}(D)$ is equal to

$$
6 \cdot k_{n}^{3}=3^{n}-3 \cdot 2^{n}+3
$$

If $Y_{\varphi_{1}}, Y_{\varphi_{2}}, Y_{\varphi_{3}}, Y_{\varphi_{4}}$ are any four elements of partitioning of $X$ and $\bar{\beta}=\left(Y_{\varphi_{1}} \times T_{1}\right) \cup\left(Y_{\varphi_{2}} \times T_{2}\right) \cup\left(Y_{\varphi_{3}} \times T_{3}\right) \cup\left(Y_{\varphi_{4}} \times T_{4}\right)$ where $T_{1}, T_{2}, T_{3}, T_{4}$ are pairwise different elements of $D$, then the number of different binary relations $\bar{\beta}$ of semigroup $B_{X}(D)$ is equal to

$$
24 \cdot k_{n}^{4}=4^{n}-4 \cdot 3^{n}+3 \cdot 2^{n}-4
$$

Let $\alpha \in B_{0}$. Quasinormal represantation of $\alpha$ has form

$$
\alpha=\left(Y_{3}^{\alpha} \times Z_{3}\right) \cup\left(Y_{2}^{\alpha} \times Z_{2}\right) \cup\left(Y_{1}^{\alpha} \times Z_{1}\right) \cup\left(Y_{0}^{\alpha} \times \breve{D}\right)
$$

where $Y_{3}^{\alpha}, Y_{2}^{\alpha}, Y_{1}^{\alpha} \notin\{\varnothing\}$. Also, $Y_{3}^{\alpha}, Y_{2}^{\alpha}, Y_{1}^{\alpha}$ or $Y_{3}^{\alpha}, Y_{2}^{\alpha}, Y_{1}^{\alpha}, Y_{0}^{\alpha}$ are partitioning of $X$ for $|X| \geq 4$. By using Equations (2.3) and (2.4) we obtain

$$
\left|B_{0}\right|=4^{n}-3^{n+1}+3 \cdot 2^{n}-1
$$

Let $\alpha \in \tilde{B}_{32}$. Quasinormal represantation of $\alpha$ has form $\alpha=\left(Y_{3}^{\alpha} \times Z_{3}\right) \cup\left(Y_{2}^{\alpha} \times Z_{2}\right)$ where $Y_{3}^{\alpha}, Y_{2}^{\alpha} \notin\{\varnothing\}$. Also, $Y_{3}^{\alpha}, Y_{2}^{\alpha}$ are partitioning of $X$. By using (2.2) we obtain 


$$
\left|\tilde{B}_{32}\right|=2^{n}-2
$$

So, we have

$$
\begin{aligned}
& \left|B_{0} \cup \tilde{B}_{32} \cup\left\{\sigma_{1}\right\}\right|=4^{n}-3^{n+1}+2^{n+2}-2 \\
& \left|B_{0} \cup \tilde{B}_{32} \cup\left\{\sigma_{0}, \sigma_{1}\right\}\right|=4^{n}-3^{n+1}+2^{n+2}-1
\end{aligned}
$$

since $B_{0} \cap \tilde{B}_{32}=B_{0} \cap\left\{\sigma_{0}, \sigma_{1}\right\}=\tilde{B}_{32} \cap\left\{\sigma_{0}, \sigma_{1}\right\}=\varnothing$.

\section{Acknowledgements}

Sincere thanks to Prof. Dr. Neşet AYDIN for his valuable suggestions.

\section{References}

[1] Diasamidze, Y. and Makharadze, S. (2013) Complete Semigroups of Binary Relations. Kriter Yay Nevi, Istanbul.

[2] Diasamidze, Y., Ayd, N.N. and Erdoğan, A. (2016) Generating Set of the Complete Semigroups of Binary Relations. Applied Mathematics, 7, 98-107.

https://doi.org/10.4236/am.2016.71009

[3] Bakuridze, A., Diasamidze, Y. and Givradze, O. Generated Sets of the Complete Semigroups of Binary Relations Defined by Semilattices of the Class $\Sigma_{1}(X, 2)$. (In Press) 